\title{
THE COHOMOLOGY RING OF A FINITE GROUP SCHEME
}

\author{
GUSTAVE EFROYMSON
}

Abstract. Let $k$ be a field and let $A$ be a $k$-algebra with additional structure so that $\operatorname{Spec} A$ is a finite commutative group scheme over $k$, (so $A$ is a Hopf algebra). Let $H^{*}(A, k)$ be the Hochschild cohomology ring. In another paper, we demonstrated that if $k$ is a perfect field:

(a) $H^{\cdot}(A, k)$ is generated by $H^{1}$ and $H_{\mathrm{gym}}^{2}$.

(b) If characteristic $k=p \neq 2$, then $H^{\cdot}(A, k)$ is freely generated by $H^{1}$ and $H_{\mathrm{aym}}^{2}$.

(c) If characteristic $k=2$, then there are subspaces $V_{1}, V_{2}$ of $H^{1}$ and $V_{3}$ of $H_{\mathrm{sym}}^{2}$ such that $H^{\circ}(A, k)$ is generated by $V_{1}, V_{2}, V_{3}$ and the only relations are $f^{2}=0$ for all $f$ in $V_{1}$.

In this paper we show that if $k$ is arbitrary (a) and (b) still hold, and we use an example of Oort and Mumford to show that (c) does not hold for arbitrary $k$.

1. $H^{\cdot}(A, k)$ for $k$ a field of characteristic $p \neq 2$. Recall briefly the definition of a finite group scheme. Let $A$ be a $k$-algebra, $A$ finitely generated as a $k$-module ( $k$ is always a field in this paper). We have maps $\Delta: A \rightarrow A \otimes A, s: A \rightarrow A, \epsilon: A \rightarrow k$ satisfying the usual axioms, [2] e.g. $(\Delta \otimes 1) \circ \Delta=(1 \otimes \Delta) \circ \Delta: A \rightarrow A \otimes A \otimes A$.

We also assume $\Delta$ is commutative. This means that if $T: A \otimes A$ $\rightarrow A \otimes A$ is defined by $T(a \otimes b)=b \otimes a$, then $T \circ \Delta=\Delta$. Then Spec $A$ together with $\Delta, s, \epsilon$ is a finite group scheme. (In other words $A$ is a Hopf algebra over $k$.)

Let $K$ be an extension of $k$. Let $A_{K}=A \otimes_{k} K$. Then Spec $A_{K}$ together with maps $\Delta \otimes 1, s \otimes 1, \epsilon \otimes 1$ is a finite group scheme over $K$.

Let $H^{\cdot}(A, k)$ be the Hochschild cohomology ring [1, p. 169], with product the cup product induced by $\Delta: A \rightarrow A \otimes A$. Then $H^{\circ}(A, k)$ is a commutative graded ring; meaning if $f$ is in $H^{n}, g$ in $H^{m}$, then $f \cup g$ $=(-1)^{m n} g \cup f$.

Recall that if $C^{\cdot}(A, k)$ is the standard resolution of $k,[1$, p. 174], then $C^{2}(A, k) \cong \operatorname{Hom}_{k}\left(A^{\otimes 2}, k\right)$. We define $H_{\text {sym }}^{2}(A, k)$ as the subgroup of $H^{2}(A, k)$ generated by the standard cocycles $f$ such that $f(x, y)$ $=f(y, x)$ for all $x, y$ in $A$.

In [2] the following theorem is proved.

Received by the editors September 8, 1969.

AMS 1969 subject classifications. Primary 1450, 1390; Secondary 1820.

Key words and phrases. Finite group scheme, Hochschild cohomology ring, $H_{\mathrm{sym}}^{2}(A, k)$, Hopf algebra. 
Theorem 1 [2, P. 317, Theorem 2.2]. If $\operatorname{Spec} A$ is a commutative group scheme over a perfect field $k$, then

(a) $H^{*}(A, k)$ is generated by $H^{1}$ and $H_{\text {sym }}^{2}$.

(b) If characteristic $(k) \neq 2$, then $H^{\circ}(A, k)$ is freely generated by $H^{1}$ and $H_{\mathrm{sym}}^{2}$. Freely generated means the only relations are $f \cup g$ $=(-1)^{m n} g \cup$ f for f in $H^{n}, g$ in $H^{m}$.

(c) If characteristic $(k)=2$, then there exist subspaces $V_{1}, V_{\mathbf{2}}$, of $H^{1}$, and $V_{3}$ of $H_{\mathrm{sym}}^{2}$ so that $H^{\circ}(A, k)$ is generated by $V_{1}, V_{2}, V_{3}$ with additional relations $f \cup f=0$ for all $f$ in $V_{1}$.

Proposition 1. Let $\mathrm{Spec} A$ be a finite group scheme over a field $k$; let $K$ be an extension field of $k$. Let $A_{K}=A \otimes_{k} K$ as above.

Then there is a natural isomorphism

$$
H^{\cdot}(A, k) \otimes_{k} K \rightarrow H^{\cdot}\left(A_{K}, K\right) .
$$

Proof. This is just another form of the universal coefficient theorem. It is proven by noting that if $C .(A, k)$ is an $A$-free resolution of the $A$-module $k$, then $C .(A, k) \otimes K=C .\left(A_{K}, K\right)$ is an $A_{K}$ free resolution of the $A_{K}$ module $K$. Also we have

$$
\begin{aligned}
\operatorname{Hom}_{\boldsymbol{\Lambda}}\left(C_{n}(A, k), k\right) \otimes K & \cong \operatorname{Hom}_{\boldsymbol{\Delta} \otimes_{k} K}\left(C_{n}(A, k) \otimes K, K\right) \\
& =\operatorname{Hom}_{\boldsymbol{A}_{K}}\left(C_{n}\left(A_{K}, K\right), K\right) .
\end{aligned}
$$

Taking cohomology we get the required isomorphism of cohomology groups. One must also check that this is a homomorphism for the ring structure but this is just a matter of checking the maps induced by $\Delta$ and $\Delta \otimes 1$.

Note that we have an injection $\phi: H^{\cdot}(A, k) \rightarrow H^{\cdot}(A, k) \otimes_{k} K$ $\cong H^{\cdot}\left(A_{K}, K\right)$.

TheOREM 2. Let $\operatorname{Spec} A$ be a finite group scheme over a field $k$. Then $H^{\cdot}(A, k)$ is generated by $H^{1}$ and $H_{\mathrm{sym}}^{2}$. Moreover $H^{\cdot}(A, k)$ is freely generated by $H^{1}$ and $H_{\mathrm{sym}}^{2}$ if characteristic $(k) \neq 2$.

Proof. Let $K$ be a perfect extension of $k$. We have $\phi: H^{*}(A, k)$ $\rightarrow H^{\cdot}(A, k) \otimes K$. We identify $H^{\cdot}(A, k) \otimes K$ with $H^{\cdot}\left(A_{K}, K\right)$. Note that $H_{\text {sym }}^{2}(A, k) \otimes K$ will then be identified with $H_{\text {sym }}^{2}\left(A_{K}, K\right)$.

Let $V_{n}$ be the subspace of $H^{n}(A, k)$ generated by $H^{1}$ and $H_{\text {sym }}^{2}$. By Theorem $1, V_{n} \otimes_{k} K=H^{n}(A, k) \otimes_{k} K$ and so $V_{n}=H^{n}(A, k)$.

Now let characteristic $(k) \neq 2$. By part (b) of Theorem 1, there are no relations on $H^{1}\left(A_{K}, K\right)$ and $H_{\mathrm{sym}}^{2}\left(A_{K}, K\right)$. Since

$$
\phi: H^{\cdot}(A, k) \rightarrow H^{\cdot}\left(A_{K}, K\right)
$$

is an injection, there cannot be any relations on $H^{1}(A, k)$ and $H_{\mathrm{sym}}^{2}(A, k)$. 
2. An example for characteristic 2. In [3, p. 320], Oort and Mumford give an example of a finite group scheme which is not of "truncation type." We are interested in this example only when $p=2$, and then it becomes:

ExAmple. Let $k$ be a field of characteristic 2. Let $a \in k, a^{1 / 2} \notin k$. Let

$$
A=k[x, y] /\left(x^{4}, x^{2}-a y^{2}\right)
$$

with $\Delta: A \rightarrow A \otimes A$ defined by $\Delta x=1 \otimes x+x \otimes 1, \Delta y=1 \otimes y+y \otimes 1$. $s$ and $\epsilon$ are the obvious maps.

Then $\operatorname{Spec} A$ is a finite group scheme over $k$.

Proposition 2. If $A$ is as above, $H^{*}(A, k)$ is a commutative graded ring isomorphic to

$$
k\left[V_{1}, V_{2}, U\right] /\left(a V_{1}^{2}-V_{2}^{2}\right),
$$

$V_{1}, V_{2}$ of degree $1, U$ of degree 2.

Proof. Let $K=k\left(a^{1 / 2}\right)=k(b)$ with $b^{2}=a$. Let $A_{K}=A \otimes_{k} K$ as before and let $x^{\prime}=x \otimes 1, y^{\prime}=y \otimes 1$. Then

$$
A_{K}=K\left[x^{\prime}, y^{\prime}\right] /\left(x^{\prime 4}, x^{\prime 2}-a y^{\prime 2}\right) \cong K\left[x^{\prime}, z\right] /\left(x^{\prime 4}, z^{2}\right)
$$

where $z=x^{\prime}-b y^{\prime}$.

We will want to define resolutions $C .(A, k)$ of the $A$ module $k$ and $C$. $\left(A_{K}, K\right)$ of the $A_{K}$ module $K$. We will use primes to denote the elements of $C .\left(A_{K}, K\right)$. Let $T_{1}, T_{2}, T_{1}^{\prime}, T_{2}^{\prime}$ be of degree 1 .

We want $\partial T_{1}=x, \partial T_{2}=y, \partial T_{1}^{\prime}=x^{\prime}, \partial T_{2}^{\prime}=z$ so we can choose $T_{1}^{\prime}=T_{1} \otimes 1, T_{2}^{\prime}=T_{1} \otimes 1+T_{2} \otimes b$.

Let $S_{1}, S_{2}, S_{1}^{\prime}, S_{2}^{\prime}$ be of degree 2 . We want

$$
\partial S_{1}=x^{3} T_{1}, \quad \partial S_{2}=x T_{1}-a y T_{2}, \quad \partial S_{1}^{\prime}=x^{\prime 3} T_{1}^{\prime}, \quad \partial S_{2}^{\prime}=z T_{2}^{\prime} .
$$

Now let $C$. $(A, k)$ be the algebra which is the exterior algebra on $T_{1}$, $T_{2}$ tensored with the divided power algebra on $S_{1}, S_{2}$. Let $C .\left(A_{K}, K\right)$ be the same thing with $T_{1}^{\prime}, T_{2}^{\prime}, S_{1}^{\prime}, S_{2}^{\prime}$ replacing $T_{1}, T_{2}, S_{1}, S_{2}$ respectively. We know $C .\left(A_{K}, K\right)$ is a free $A_{K}$ resolution of $K,[2, \mathrm{p}$. 315]. Also we can let $S_{1}^{\prime}=S_{1} \otimes 1$ and $S_{2}^{\prime}=S_{2} \otimes 1+T_{1} T_{2} \otimes b$. Then it is clear that $C .(A, k) \otimes_{k} K$ can be identified with $C .\left(A_{K}, K\right)$. This means that $C .(A, k)$ is a free resolution also.

Take $C^{*}(A, k)=\operatorname{Hom}_{A}(C .(A, k), \quad k)$ and $C^{*}\left(A_{K}, K\right)=$ $\operatorname{Hom}_{A_{K}}\left(C .\left(A_{K}, K\right), K\right)$. So $C^{1}(A, k) \cong \operatorname{Hom}_{A}\left(A^{\otimes 2}, k\right) \cong \operatorname{Hom}_{k}\left(k^{\otimes 2}, k\right)$.

Let $V_{1}, V_{2}$ be the dual basis to $T_{1}, T_{2}$ under the above identification so $V_{1}\left(T_{1}\right)=1$, etc. Then $V_{1} V_{2}$ will be dual to $T_{1} T_{2}$ in $C^{2}(A, k)$. Com- 
plete the dual basis by letting $U_{1}, U_{2}$ be $k$ dual to $S_{1}, S_{2}$. Do the same for the primes. Then

$$
C \cdot\left(A_{K}, K\right) \cong K\left[V_{1}^{\prime}, V_{2}^{\prime}, U_{1}^{\prime}, U_{2}^{\prime}\right] /\left(V_{1}^{\prime 2}, V_{2}^{\prime 2}-U_{2}^{\prime}\right)
$$

with $\delta V_{i}^{\prime}=0=\delta U_{i}^{\prime}$. See [2, p. 317, Corollary].

We also have a natural map identifying $C^{\cdot}\left(A_{K}, K\right)$ with $C^{*}(A, k) \otimes K$. We have

$$
\left(\begin{array}{l}
T_{1}^{\prime} \\
T_{2}^{\prime}
\end{array}\right)=\left(\begin{array}{ll}
1 & 0 \\
1 & b
\end{array}\right)\left(\begin{array}{l}
T_{1} \otimes 1 \\
T_{2} \otimes 1
\end{array}\right) .
$$

Dualizing we get

$$
\left(\begin{array}{l}
V_{1} \otimes 1 \\
V_{2} \otimes 1
\end{array}\right)=\left(\begin{array}{ll}
1 & 1 \\
0 & b
\end{array}\right)\left(\begin{array}{l}
V_{1}^{\prime} \\
V_{2}^{\prime}
\end{array}\right)
$$

Also

$$
\left(\begin{array}{c}
S_{1}^{\prime} \\
S_{2}^{\prime} \\
T_{1}^{\prime} T_{2}^{\prime}
\end{array}\right)=\left(\begin{array}{lll}
1 & 0 & 0 \\
0 & 1 & b \\
0 & 0 & b
\end{array}\right)\left(\begin{array}{c}
S_{1} \otimes 1 \\
S_{2} \otimes 1 \\
T_{1} T_{2} \otimes 1
\end{array}\right)
$$

induces

$$
\left(\begin{array}{c}
U_{1} \otimes 1 \\
U_{2} \otimes 1 \\
V_{1} V_{2} \otimes 1
\end{array}\right)=\left(\begin{array}{lll}
1 & 0 & 0 \\
0 & 1 & 0 \\
0 & b & b
\end{array}\right)\left(\begin{array}{c}
U_{1}^{\prime} \\
U_{2}^{\prime} \\
V_{1}^{\prime} V_{2}^{\prime}
\end{array}\right) .
$$

On the cohomology level, we have $V_{1}^{\prime 2}=0, V_{2}^{\prime 2}=U_{2}^{\prime}$. However $V_{1} \otimes 1=V_{1}^{\prime}+V_{2}^{\prime}$ which implies $V_{1}^{2} \otimes 1=U_{2}^{\prime}$. Similarly $V_{2} \otimes 1=b V_{2}^{\prime}$ implies $V_{2}^{2} \otimes 1=b^{2} U_{2}^{\prime}=a U_{2}^{\prime}$. Thus $a V_{1}^{2}=V_{2}^{2}$. Moreover $V_{1}^{2}=U_{2}$. Let $U=U_{1}$. Our result follows since $H^{\cdot}(A, k) \otimes_{k} K=H^{\cdot}\left(A_{K}, K\right)$ and so no more relations are possible.

\section{REFERENCES}

1. H. Cartan and S. Eilenberg, Homological algebra, Princeton Univ. Press, Princeton, N. J., 1956. MR 17, 1040.

2. G. Efroymson, Certain cohomology rings of finite and formal group schemes, Trans. Amer. Math. Soc. 145 (1969), 309-322.

3. F. Oort and D. Mumford, Deformations and liftings of finite commutative group schemes, Invent. Math. 5 (1968), 317-344. MR 37 \#4085.

University of New Mexico, Albuquerque, New Mexico 87106 